\title{
Prototipo web para el procesamiento de registros de pacientes potencialmente infectados con tuberculosis en la Huasteca Hidalguense
}

\section{Web prototype for the processing of records of patients potentially infected with tuberculosis in the Huasteca Hidalguense}

\author{
SALAZAR-CASANOVA, Hermes $\dagger^{*}$, MENDOZA-SAN JUAN, Luis Alberto y FELIPE-REDONDO, \\ Ana María
}

Universidad Tecnológica de la Huasteca Hidalguense

ID $1^{\text {er }}$ Autor: Hermes, Salazar-Casanova / ORC ID: 0000-0002-8444-6186, Researcher ID Thomson: O-6647-2018, CVU CONACYT ID: 926456

ID $1^{\text {er }}$ Coautor: Luis Alberto, Mendoza-San Juan / ORC ID: 0000-0002-7186-0177, Researcher ID Thomson: O-6650-2018, CVU CONACYT ID: 344988

ID $2{ }^{\text {do }}$ Coautor: Ana María, Felipe-Redondo / ORC ID: 0000-0002-8579-6532, Researcher ID Thomson: O-7111-2018, CVU CONACYT ID: 835952

DOI: $10.35429 /$ JCA.2019.12.3.1.7

Recibido Julio 30, 2019; Aceptado Noviembre 30, 2019

\begin{abstract}
Resumen
El desarrollo de un prototipo web para el registro de muestras de pacientes potencialmente infectados con tuberculosis es de gran importancia debido a que la información generada a partir de los resultados de laboratorio debe ser transmitida de manera rápida, veraz e íntegra a las unidades médicas solicitantes con la finalidad de brindar un tratamiento específico acorde al diagnóstico obtenido en los muestreos previamente analizados. Construir una plataforma web mediante la metodología de Ingeniería Web (IWeb) permite optimizar el envío de los resultados de las pruebas para que puedan ser consultados desde cualquier entidad autorizada por la Secretaría de Salud del Estado de Hidalgo en tiempo real, de forma inmediata y sin intermediarios para reducir los trámites burocráticos que resultan tardados, además de poco confiables. Este proyecto nace como una solución ante la problemática que se presenta durante los servicios de atención a los pacientes, ya que en esta etapa es donde se presenta la mayor cantidad de dificultades para entregar resultados de forma inmediata, acción que contribuye a que la diagnosis de la enfermedad se lleve a cabo de manera tardía y propiciando nuevos contagios de este padecimiento.
\end{abstract}

Prototipo, Web, Tuberculosis

\begin{abstract}
The development of a web prototype for the registration of samples of patients potentially infected with tuberculosis is of great importance because the information generated from the laboratory results must be transmitted in a rapid, truthful and complete manner to the medical units that request them with the purpose of providing a specific treatment according to the diagnosis obtained in the previously analyzed samples. Building a web platform through the Web Engineering methodology allows optimizing the sending of test results so that they can be consulted from any entity authorized by the Secretary of Health of the State of Hidalgo in real time, immediately and without intermediaries to reduce bureaucratic procedures that are delayed, in addition to unreliable. This project was born as a solution to the problems that arise during patient care services, since at this stage is the most difficult to deliver results immediately, an action that contributes to the diagnosis of the disease is carried out late and causing new infections of this disease.
\end{abstract}

Citación: SALAZAR-CASANOVA, Hermes, MENDOZA-SAN JUAN, Luis Alberto y FELIPE-REDONDO, Ana María. Prototipo web para el procesamiento de registros de pacientes potencialmente infectados con tuberculosis en la Huasteca Hidalguense. Revista de Cómputo Aplicado. 2019, 3-12: 1-7

\footnotetext{
* Correspondencia al Autor (Correo electrónico: hermes.salazar@uthh.edu.mx)

$\uparrow$ Investigador contribuyendo como primer autor.
} 


\section{Introducción.}

La tuberculosis (TB), representa un importante problema emergente de salud pública a nivel mundial, ya que se considera actualmente la enfermedad infecciosa más letal, además de tener importantes consecuencias económicas y sociales. (Cacho, 2015).

La prevención y control de la tuberculosis representan un actuar permanente de todos aquellos trabajadores de la salud, inmersos dentro de las áreas de competencia de la salud pública. Por lo anterior, resulta necesaria la interacción sectorial y multidisciplinaria en los ámbitos tanto públicos como privados, para avanzar en los retos y desafíos actuales y futuros ante el control de esta milenaria enfermedad, que aún representa un problema de salud pública en México. (Secretaría de Salud, 2014).

El proyecto denominado "Prototipo web para el procesamiento de registros de pacientes potencialmente infectados con tuberculosis en la Huasteca Hidalguense" incluye el desarrollo de una aplicación web que surge a partir de la problemática presentada por los laboratoristas de la Jurisdicción Sanitaria de Huejutla de Reyes, Hidalgo debido a que no cuentan con un control sistematizado de registro para las personas con este padecimiento.

El prototipo se encuentra elaborado en un entorno web, específicamente se empleó el Framework Laravel 5.8.28 que reduce en gran medida los tiempos de desarrollo y mantenimiento, otro beneficio de emplear esta herramienta es que se encuentra basado en el patrón de diseño Modelo Vista Controlador (MVC) que ofrece una adecuada organización del proyecto.

La aplicación permite capturar información referente a los distintos centros de salud que existen en la Huasteca Hidalguense, para poder relacionarlos con sus municipios, del mismo modo pueden registrarse doctores enfermeras y pacientes para que al momento de dar de alta una muestra, siempre exista una trazabilidad que permita en todo momento saber el estado de la muestra realizada.
Este artículo se encuentra dividido en las siguientes secciones: Problemática que describe las diferentes situaciones y dificultades que motivaron la realización de esta investigación, el siguiente apartado es la justificación misma que menciona cuales son las soluciones que se proponen con la implementación de este proyecto.

A continuación se enuncia el objetivo que se desea conseguir; dentro de los Fundamentos Teóricos se incluyen la conceptualización necesaria que ubica al lector para facilitar la comprensión del tema abordado. La metodología enuncia las diferentes etapas a seguir en el desarrollo del prototipo; el desarrollo describe las tareas realizadas en cada una de las fases. Finalmente, se incluyen las conclusiones que resultaron en este estudio

\section{Problemática}

Actualmente la Jurisdicción Sanitaria ubicada en la ciudad de Huejutla de Reyes, Hidalgo no cuenta con una aplicación que permita registrar las muestras de personas que son candidatos a padecer tuberculosis. Esta información debe ser transmitida a diferentes entidades de dicha dependencia mediante trámites burocráticos que resultan tardados y poco confiables.

Aunado a esto, los laboratoristas realizan los registros de las pruebas de manera manual para después almacenarlos en archiveros, lo que aumenta la posibilidad de extravío de información y desorganización de archivos, mismos que resultan en un inefectivo procesamiento de resultados, retrasando así el tiempo de respuesta y ocasionando que las personas no reciban un tratamiento específico y en el tiempo adecuado.

A su vez, y debido a la naturaleza no sistematizada de llevar a cabo el tratamiento de la información, conlleva a que el personal de salubridad no cuente con el elemento de trazabilidad para el rastreo de pacientes declarados positivos con tuberculosis, lo que aumenta las posibilidades de que se presenten más contagios por parte de los enfermos con sus familiares o personas con las que interactúa de manera directa, dificultando los esfuerzos por controlar la propagación de esta enfermedad en la región Huasteca del Estado de Hidalgo. 


\section{Justificación}

Las Tecnologías de Información y Comunicación (TIC), junto con las metodologías avanzadas de procesamiento de la información han evolucionado significativamente, influyendo activamente en nuestra sociedad. En salud, este impacto estaba orientado a la optimización de procesos, al incremento de productividad y la gestión financiera, en lo que conocemos como la era de los "sistemas de información hospitalarios".

Sin embargo, al entender el flujo de trabajo y contexto clínico como una serie de procesos complejos en los que el paciente y su interacción con el sistema de salud son el centro de la escena, estos sistemas evolucionaron hacia sistemas de información en salud, donde la captura, intercambio, almacenamiento, acceso y gestión de información clínica adquieren un rol fundamental, con el objetivo de contribuir a la calidad y eficiencia de la atención de salud, mejorar la accesibilidad a sus servicios y el conocimiento médico. (Haux, 2006)

Las TIC también permiten un tratamiento sistemático de datos, información y conocimiento en el entorno sanitario y contribuyen considerablemente al progreso de las ciencias de la salud. Entre los beneficios de implementar TIC se puede encontrar que favorecen la continuidad en el cuidado de los pacientes gracias a que mejoran la comunicación del equipo de salud y el acceso ubicuo a la información clínica. (Bright, Dhurjati, Bristow, Bastian, Coeytaux, 2012)

El propósito en esta investigación es desarrollar un prototipo que facilite la transferencia de información de manera rápida y oportuna para que pueda ser accesada en tiempo real desde cualquier entidad autorizada por la Jurisdicción Sanitaria de la región Huasteca Hidalguense.

Para resolver esta situación se determinó que el mejor entorno para su operación fuera internet debido a que por su naturaleza, es un recurso que actualmente es utilizado por la mayoría de las personas para la transmisión de información a cualquier lugar del planeta en tiempos reducidos de tiempo.
A su vez, permite que el empleo de la aplicación pueda darse desde dispositivos móviles o computadoras personales, agilizando los procesos de captura de datos que pueden traducirse en resultados inmediatos y por ende la determinación de una atención y tratamiento adecuado y oportuno para los potenciales pacientes infectados con tuberculosis.

\section{Objetivo}

Desarrollar un prototipo web apoyado en la metodología IWeb para facilitar una herramienta sistematizada de registro de potenciales pacientes con tuberculosis en la región Huasteca Hidalguense.

\section{Fundamentos teóricos}

En esta sección se enuncia la descripción detallada de la conceptualización de los elementos presentes en esta investigación para facilitar la comprensión de los términos empleados en este estudio.

\section{Tuberculosis}

La tuberculosis es causada por Mycobacterium Tuberculosis, una bacteria que casi siempre afecta a los pulmones. Se trata de una afección curable y que se puede prevenir. La infección se transmite de persona a persona a través del aire. Cuando un enfermo de tuberculosis pulmonar tose, estornuda o escupe, expulsa bacilos tuberculosos al aire. Basta con que una persona inhale unos pocos bacilos para quedar infectada. Se calcula que una cuarta parte de la población mundial tiene tuberculosis latente, término este aplicado a las personas infectadas por el bacilo pero que aún no han enfermado ni pueden transmitir la infección. (OMS, 2018).

A pesar de los avances tecnológicos y grandes esfuerzos del personal de salud, la tuberculosis sigue siendo un grave problema de salud pública.

Cada segundo, en el mundo se produce una nueva infección por el bacilo de la TB. De acuerdo con las cifras reportadas por la OMS, en el año 2015 se presentó un nuevo enfermo cada 3 segundos y otro más murió cada 18 segundos. (Orozco, Acosta, Bravo, Martínez, Enríquez, Espinoza, Durán, Orozco, 2018). 
Entre 2000 y 2011, las infecciones respiratorias agudas (IRA) se mantuvieron como la principal causa de enfermedad en nuestro país. $\mathrm{Su}$ tasa de incidencia pasó de 29,441.34 a 23,672.84 casos/100 mil. (SIAVE, 2012).

\section{Tuberculosis en México}

La tuberculosis en México no se ha logrado erradicar; se ha reducido el número de muertes en más de 45 por ciento, pero la incidencia se ha mantenido, con una ligera disminución en el número de casos.

Para 2009, en nuestro país según un reporte editado por el Comité Nacional de Lucha contra la tuberculosis y Enfermedades del Aparato Respiratorio mueren 5 mil mexicanos al año por esta enfermedad, se conocen cerca de 30 mil casos nuevos por año y se infectan otros 1000 diariamente.

Aproximadamente el $84 \%$ de los pacientes que ingresan a tratamiento se curan, $2.5 \%$ muere y $13.5 \%$ se registra como fracaso, abandono del tratamiento. Estas proporciones varían de acuerdo con la localización de la enfermedad. (Orozco, Nesbitt, González, 2009)

Estudios más recientes calculan que se presentan de 2,000 a 2,500 muertes por año, y según cifras de la Secretaría de Salud, se reportan más de 19 mil casos nuevos anualmente. (Castillo, 2018).

En Hidalgo, la tuberculosis pulmonar continúa siendo un problema de salud pública, al estratificar en el 2016 los riesgos municipales con la mortalidad acumulada por tuberculosis pulmonar 2009-2014, la morbilidad acumulada por tuberculosis pulmonar 2010-2015, cobertura de detección 2015, porcentaje de tuberculosis fármaco resistente y porcentaje de fracasos, recaídas y abandono de tratamiento, se identifica riesgo alto en 45 municipios: 12 ubicados en la región de La Huasteca, 3 en la Sierra de Tenango, 8 en la Sierra Alta, 2 en la Sierra Baja, 3 en la Sierra Gorda, 3 en el Valle de Tulancingo, 1 en la Comarca Minera, 1 en Altiplano y 12 en el Valle del Mezquital.

Con riesgo medio se reconocen 28 municipios que representan el 33.3\% del total y los 11 municipios restantes se clasifican con riesgo bajo. (Secretaría de Salud de Hidalgo, 2016).

\section{Aplicaciones web}

En la Ingeniería de software se denomina aplicación web a aquellas aplicaciones que los usuarios pueden utilizar accediendo a un Servidor web a través de Internet o de una intranet mediante un navegador. En otras palabras, es una aplicación (Software) que se codifica en un lenguaje soportado por los navegadores web en la que se confía la ejecución al navegador.

Las aplicaciones web tienen varias ventajas sobre las aplicaciones tradicionales, entre ellas está la compatibilidad; ya que utilizan el navegador del cliente como interfaz de usuario, debido a que el lenguaje HTML garantiza la compatibilidad en distingas plataformas.

El segundo elemento es la accesibilidad, porque el acceso remoto es uno de sus prerrequisitos de diseño, los dispositivos móviles están generalmente soportados. (Palacios, Puente).

\section{Metodología}

Para la realización de este proyecto se eligió la metodología de Ingeniería Web (IWeb) debido a que establece y utiliza principios de ingeniería y de gestión con enfoques sistemáticos $\mathrm{y}$ disciplinados para el desarrollo, empleo y mantenimiento de aplicaciones basadas en Web.

La estructura de la IWeb se compone de 6 fases: Comunicación, planeación, modelado, construcción, despliegue, documentación (Figura 1).

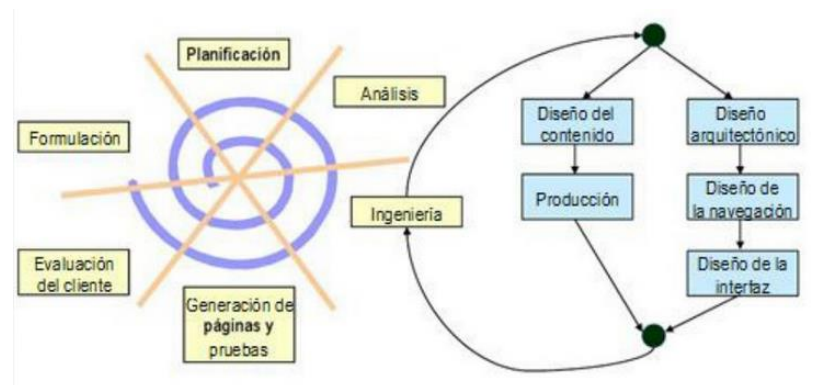

Figura 1 Fases de la metodología IWeb

Dentro del proceso web la comunicación se identifican los participantes donde se definen los problemas que se han de resolver mediante la Web App en base a los requisitos. 
A continuación, se crea el plan del proyecto que consiste de una definición de tareas y un calendario de plazos respecto al periodo usualmente medido en semanas proyectando para el desarrollo del mismo.

Las labores convencionales de diseño de ingeniería de software se adaptan el desarrollo de la aplicación, se mezclan y luego se funden en la actividad de modelado web. Se realizan modelados de diseño que definan requisitos y al mismo tiempo representen una Web App que satisfaga al usuario final.

Las herramientas y la tecnología web se aplican para realizar la construcción que se ha modelado. Una vez que se construye el incremento se debe asegurar que se descubran los errores en el diseño es decir contenido, arquitectura, interfaz gráfica de usuario (IGU), navegación y pruebas adicionales.

El prototipo se entrega a los usuarios finales y después comienza un periodo de evaluación. La retroalimentación acerca de la evaluación se presenta al equipo desarrollador y el incremento se modifica conforme se requiera. (Pressman, 2005).

\section{Desarrollo}

En la primera etapa se efectuó la comunicación con los usuarios y para ello se realizó una entrevista para recabar información relacionada con los requerimientos. Se identificaron los tres participantes de la Jurisdicción Sanitaria de Huejutla de Reyes, Hidalgo que serán los encargados de utilizar la aplicación web. Entre ellos se encuentran los doctores, laboratoristas y enfermeras que podrán ejecutar funciones diferentes y específicas de cada puesto.

Además, entre los aspectos más importantes, se abordaron temas referentes al aspecto deseado para la Web App, el esquema de colores que se incluirían en el diseño del prototipo, características especiales deseadas en la interfaz gráfica de cada usuario, así como las fechas de culminación de proyecto. Dentro de la etapa de planeación se obtuvo los tiempos de cada una de las tareas que se realizaron en el plazo de 14 semanas, para ello se creó un plan para el desarrollo de este proyecto. El plan consiste de una definición de tareas y un calendario de plazos expresados en el Diagrama de Gantt (Figura 2).

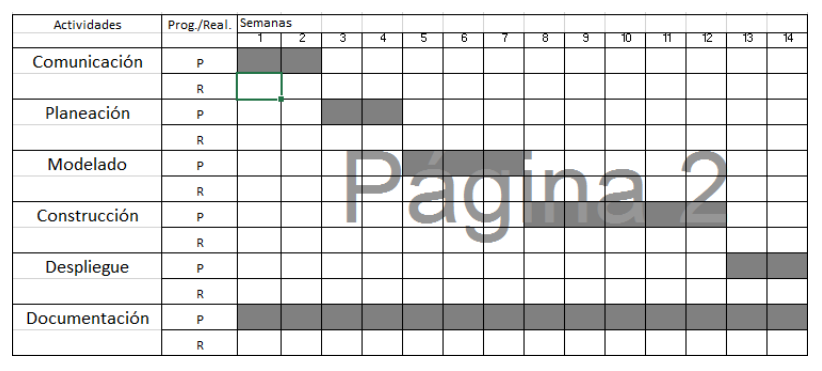

Figura 2 Planeación de actividades realizadas

Una vez establecidos los requerimientos específicos para cada tipo de usuario, se procedió al diseño de bocetos de la interfaz gráfica de usuario. Cada bosquejo se apegó de manera precisa a las características solicitadas, incluyendo elementos de usabilidad para podar ofrecer una operación intuitiva.

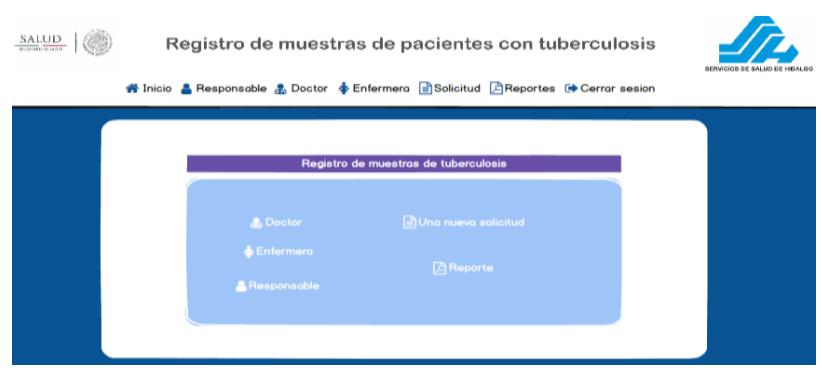

Figura 3 Boceto de la Página de Inicio del prototipo web

En la etapa de construcción, los bocetos fueron utilizados en los modelados para realizar los diseños de manera definitiva y garantizar que éstos sean óptimos para cada módulo de la Web App.

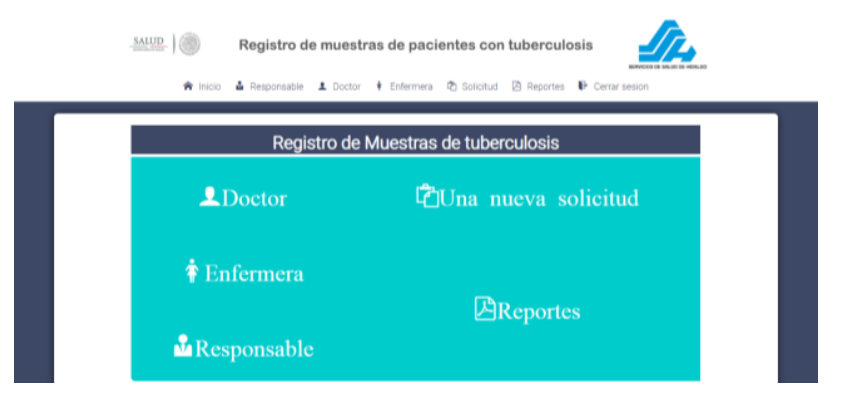

Figura 4 Instantánea de la Página de Inicio del prototipo web

En la última fase se generaron pruebas de validaciones, pruebas de su funcionalidad en los registros, pruebas de $\log$ in, se revisaron los errores que se presentaron durante la ejecución de la aplicación y se corrigieron los desperfectos, también fueron atendidas todas las observaciones y aportaciones efectuadas por el personal de la Jurisdicción Sanitaria de Huejutla de Reyes, Hidalgo. 
Por último, dentro de la etapa de documentación se diseñaron los manuales técnico, de usuario, así como la guía de estilo de la IGU.

\section{Resultados}

El primer paso tomado en este proyecto fue el acercamiento entre docentes del Cuerpo Académico de Tecnologías de la Información de la Universidad Tecnológica de la Huasteca Hidalguense con el personal de la Jurisdicción Sanitaria de Huejutla de Reyes, Hidalgo con el propósito de intercambiar información importante sobre los requerimientos y problemática a solucionar en el registro de pacientes potencialmente infectados con tuberculosis.

La propuesta de solución obtenida a partir de dicha reunión fue un prototipo web que satisficiera la necesidad de agilizar el registro de pacientes, muestras y usuarios, reducir los tiempos en el procesamiento y transferencia de información para mejorar la atención de las personas y ayudar al diagnóstico y tratamiento de las mismas.

En reuniones de Cuerpo Académico y colaborativas, se definió la metodología de desarrollo de software a emplear en el desarrollo de la aplicación web. Con esta forma de trabajo se definieron las actividades a realizar, los tiempos asignados a cada una de ellas y la asignación de compromisos por parte de cada integrante del equipo desarrollador. Se estableció un plan de trabajo dividido en semanas basado en la Ingeniería Web que controló y permitió monitorear cada avance ejecutado en la realización de las diferentes tareas fijadas en cada etapa: Comunicación, planeación, modelado, construcción, despliegue y documentación.

Primeramente se establecieron durante una entrevista con el personal de la Jurisdicción Sanitaria los requerimientos y elementos de usabilidad, posteriormente se definieron las factibilidades técnicas y de software a emplear para la construcción de la Web App. El diseño de bocetos en la etapa de modelado fue de gran utilidad para el desarrollo de los diferentes módulos contemplados en la aplicación web, debido a que se aplicaron todas las características establecidas en la guía de estilo de la IGU.
Para finalizar, se realizaron las pruebas de validación, registro, log in y se atendieron todas las observaciones y aportaciones de usabilidad realizadas por los usuarios finales, resolviendo las áreas de oportunidad.

\section{Conclusiones}

En la actualidad, el Prototipo web para el procesamiento de registros de pacientes potencialmente infectados con tuberculosis en la Huasteca Hidalguense se encuentra se encuentra terminada en su primera etapa.

$\mathrm{Su}$ funcionamiento aún se encuentra limitado al personal de la Jurisdicción Sanitaria de Huejutla de Reyes, Hidalgo de manera local. Dicha autoridad sanitaria está gestionando los recursos necesarios para que este proyecto pueda alojarse en la web y pueda ser utilizado, en primera instancia, dentro de la jurisdicción número 10.

Esta dependencia cuenta con un sistema de registro de pacientes con tuberculosis integrado en la página web de la Secretaría de Salud de Hidalgo, pero para realizar este procedimiento se deben llenar formatos de manera manual, después se sigue un proceso burocrático que causa demoras en la entrega de resultados y por ende en la atención y tratamiento de los pacientes.

Con la culminación de la primera etapa del prototipo, se determina que este proyecto tiene potencial para poder solventar la problemática referente al procesamiento $\mathrm{y}$ transferencia de información de expedientes clínicos y muestras de los diferentes centros de salud de la Jurisdicción Sanitaria número 10 de Hidalgo.

Como propuesta, se pretende aplicar las Normas Oficiales Mexicanas dirigidas al procesamiento de información clínica de pacientes a través de la web a este proyecto para que pueda ser compatible en su totalidad con la plataforma web de la Secretaría de Salud de Hidalgo.

\section{Agradecimientos}

Al personal de la Jurisdicción Sanitaria número 10 ubicada en la ciudad de Huejutla de Reyes, Hidalgo en especial al Dr. Esteban Quevedo Moraila, responsable del área de investigación. 


\section{Referencias}

Bright TJ, Wong A, Dhurjati R, Bristow E, Bastian L, Coeytaux RR, et al. Effect of clinical decision-support systems: a systematic review. Ann Intern Med. 2012.

Cacho-Carranza Y. Epidemiología de la tuberculosis en México, Agencia informativa Conacyt. (2015) México, DF. Disponible en:http://conacytprensa.mx/index.php/ciencia/s alud/1103-epidemiologia-de-la-tuberculosis-enmexico

Castillo Rodal, Antonia Isabel. Tuberculosis, enfermedad infecciosa más letal en el mundo. Boletín UNAM-DGCS-187bis. Ciudad Universitaria. Marzo de 2018.

Haux, R. Health information systems - past, present, future. Int J Med Inform. 2006.

OMS, Organización Mundial de la Salud. Tuberculosis datos y cifras. 2018. Disponible en: https://www.who.int/es/news-room/factsheets/detail/tuberculosis

Orozco-Andrade, Isaías, Acosta-Loya, Jesús Aníbal, Bravo-Rodríguez, Gregorio, MartínezLozano, Fabricio N., Enríquez-Porras, Alejandra, Espinoza-Hernández, Manuel E., Durán-Peña, Omar A., Orozco-Béjar, Isaías A. Epidemiología de tuberculosis pulmonar en población migrante. Neumol Cir Torax, Vol. 77 - Núm. 2:125-131, Abril-junio 2018.

Orozco, I., Nesbitt, C., González, S. Tuberculosis en pediatría: epidemiología. Revista de Enfermedades infecciosas en Pediatría 2009.

Palacios Hielscher, Rafael, Puente Águeda, Cristina. DESARROLLO DE APLICACIONES WEB. Disponible en: https://www.iit.comillas.edu/palacios/cursoApp Web/cap01.pdf

Pressman, Roger S. Ingeniería de software, un enfoque práctico. Sexta edición. Mc Graw Hill. 2005. ISBN: 970-10-5473-3.

Secretaría de Salud. Prevención y Control de la Tuberculosis. Programa Sectorial de Salud 2013-2018. Primera edición, 2014.
Secretaría de Salud de Hidalgo. Programa Sectorial de Salud 2016-2022. Disponible en: https://s-

salud.hidalgo.gob.mx/contenido/informacion/p ublicaciones/Programa\%20Sectorial\%20de\%20 Salud.pdf

SIAVE. Información epidemiológica sobre morbilidad. Anuario 2011 Versión Ejecutiva. México: Secretaría de Salud; 2012. Disponible en:

http://www.epidemiologia.salud.gob.mx/doctos /infoepid/publicaciones/2012/ver_ejecutiva_ 2011.pdf 